\title{
CYTOKINES AND ACUTE-PHASE SERUM PROTEINS AS MARKERS OF INFLAMMATORY REGRESSION DURING PULMONARY TUBERCULOSIS TREATMENT
}

Thesis: E. Peresi submitted this thesis for her Master's in Tropical Diseases at Botucatu School of Medicine, São Paulo State University, UNESP, Botucatu, São Paulo, Brazil, 2007.

Advisor: Professor Jussara Marcondes Machado

ABSTRACT: Tuberculosis is still increasing and was declared a worldwide sanitary emergency by the World Health Organization (WHO) in 1995. Its control is difficult due to long treatment duration and lack of markers of treatment success or failure. Cytokines such as IFN- $\gamma$ and TNF- $\alpha$, a central factor in immune response against Mycobacterium tuberculosis, are responsible for the interaction between $T$ lymphocytes and the infected macrophage and are also produced during this interaction. As proinflammatory cytokines have a close relationship with mycobacteria clearance, in fact even preceding it, they could be used as markers for inflammatory activity and response to treatment. Proinflammatory cytokines act in the liver and stimulate a strong local and systemic acute-phase response as a result of homeostatic and physiological responses also induced by them. Acute-phase proteins produced by cytokine activity are useful diagnostic markers that could also be used to monitor treatment response as they can be serially quantified. The objective of this study was to evaluate IFN- $\gamma$, TNF- $\alpha$, IL-10 and TGF- $\beta$ production in supernatant of peripheral blood mononuclear cell (PBMC) and monocyte (MO) cultures, as well as serum acute-phase response through total protein, albumin, globulin, C-reactive protein (CRP), a-1-acid glycoprotein (AGP), and erythrocyte sedimentation rate (ESR) as regression markers of inflammatory response during pulmonary tuberculosis treatment. Twenty blood donors (G1) from the Blood Bank at Botucatu School of Medicine's University Hospital (BSM-UH) were evaluated once and 28 pulmonary tuberculosis patients (G2): 13 from BSM-UH and 15 from the Bauru State Health Secretariat. Patients were evaluated at three moments of treatment: before (M1), at three months (M2), and at the end (M3). Cytokines were determined in $20 \mathrm{ml}$ of peripheral blood (ELISA), with or without activation: lipopolysaccharide (LPS) for MO culture and phytohemagglutinin (PHA) for PBMC culture. Acute-phase protein behavior in $\mathrm{G} 2$ throughout treatment was: Globulins: M1>M2, M1>M3 ( $\rho<0.001)$; CRP: M1>M2>M3 $(\rho<0.001)$; AGP for men: M1>M2, M1>M3 $(\rho<0.001)$; ESR for men: M1>M2, M1>M3 $(\rho<0.0016)$ and for women: $M 1>M 2(\rho<0.025)$. Comparison between cytokine levels found in supernatant of MO and PBMC cultures, with and without stimulus, in $\mathrm{G} 1$ and $\mathrm{G} 2$ during treatment showed: TNF- $\alpha$ (with/without LPS) at M1: G2>G1; at M2: G2>G1 $(\rho<0.001$ ); (without LPS) at M3: G2>G1 $(\rho<0.001)$, (with LPS) at M3: G2>G1 $(\rho<0.028)$; IFN-Y (with and without PHA) at M1: G2>G1; at M2: G2>G1 ( $\rho<0.001)$; IL-10 (with and without LPS) at M1: G2>G1; at M2: G2>G1; at M3: G2>G1 $(\rho<0.001)$; TGF- $\beta$ (with and without LPS) at M1: G2>G1; at M2: G2>G1 $(\rho<0.001)$, (without LPS) at M3: G2>G1 
$(\rho<0.001)$. In $\mathrm{G} 2$, all cytokines in supernatant of $\mathrm{MO}$ and PBMC cultures, with and without stimulus, showed: M1>M2>M3 ( $\rho<0.01)$. Levels of globulins, CRP, AGP, and ESR in patients with pulmonary tuberculosis before treatment (M1) were significantly higher than reference values, suggesting their use as diagnostic markers and indicators of treatment. The CRP decreasing values along treatment could be taken as a marker of the regression of inflammatory process and of response to treatment in patients with pulmonary tuberculosis. Regarding cytokines, there was significant increase in TNF- $\alpha$, IFN- $\gamma$, IL-10, and TGF- $\beta$ levels before and at three months treatment, with and without stimulus; in TNF- $\alpha$ and IL-10 levels, with and without stimulus, as well as in TGF- $\beta$ levels without stimulus at six months. Patients had higher levels of all studied cytokines than controls before treatment, and these values decreased along treatment. In this study, pulmonary tuberculosis patients showed a Th0 cytokine profile before treatment, with the production of both Th1 (IFN- $\gamma$ ) and Th2 (IL-10) cytokines, in addition to TNF- $\alpha$ inflammatory and TGF- $\beta$ regulatory and fibrosis-inducer cytokines. At the end of treatment, all had evolved to Th2 profile, probably in an attempt to reduce the harmful effects of the proinflammatory activity of the Th1 cytokine profile and of the still above-normal levels of TNF- $\alpha$. The high levels of TGF- $\beta$, also found in these patients, are related to its important role in the extracellular matrix deposition and fibrosis induction that characterize tuberculosis healing process. IFN- $\gamma$ was the only cytokine reaching normal levels at the end of treatment, which suggests its use as a marker of response to treatment.

KEY WORDS: acute-phase response, cytokines, Mycobacterium tuberculosis, tuberculosis, treatment.

CORRESPONDENCE TO:

ELIANA PERESI, Departamento de Doenças Tropicais e Diagnóstico por Imagem, Faculdade de Medicina de Botucatu, UNESP, Distrito de Rubião Junior, s/n, 18618000, Botucatu, SP, Brasil. Phone: 551438116212.

Email: elianaperesi@yahoo.com.br. 